\title{
A Facile and Practical $p$-Toluenesulfonic Acid Catalyzed Route to Dicoumarols Containing an Aroyl group
}

\author{
Saeed Khodabakhshi, ${ }^{\mathrm{a}, \boldsymbol{}}$ Bahador Karami, ${ }^{\mathrm{b}}$ Khalil Eskandari ${ }^{\mathrm{b}, \star}$ and Alimorad Rashidi ${ }^{\mathrm{a}}$ \\ ${ }^{a}$ Nanotechnology Research Center, Research Institute of Petroleum Industry, Tehran, Iran. \\ ${ }^{b}$ Department of Chemistry, Yasouj University, P.O. Box 353, Yasouj, 75918-74831, Iran.
}

Received 6 May 2014, revised 29 January 2015, accepted 2 February 2015.

\begin{abstract}
New and known dicoumarols may be efficiently synthesized employing $p$-toluenesulfonic acid ( $p$-TSA) as a solid acid catalyst from the reaction of 4-hydroxycoumarin with aryl glyoxal in water. This method offers direct access to structurally diverse coumarin derivatives in moderate to good yields (up to 65\%). A total of five new compounds were synthesized.
\end{abstract}

KEYWORDS

Dicoumarol, $p$-toluenesulfonic acid, aryl glyoxal, 4-hydroxycoumarin.

\section{Introduction}

Among the analogues of vitamin $\mathrm{K}$ antagonists, dicoumarol, which may be considered as bridge substituted dimers of 4-hydroxycoumarin, is a naturally occurring anticoagulant. ${ }^{1}$ This compound is used for the prevention and treatment of thrombosis. Furthermore, dicoumarol derivatives exhibit bioactivity as inhibitor of reductases. ${ }^{2}$ The chemistry of coumarin derivatives has recently gained much attention from chemists owing to some interesting biological properties. ${ }^{3-5}$

Dicoumarol was firstly discovered in moldy wet sweet-clover hay subsequent to which several methods have been reported for the development of its chemistry and synthesis of derivatives. Traditionally, the most popular strategies towards the synthesis of dicoumarols start from salicylaldehyde and formaldehyde $^{6}$ and involve the biosynthesis of dicoumarol using micro-organisms such as Penicillium jenseni, ${ }^{7}$ or require the Knoevenagel condensation of 4-hydroxycoumarins with carbonyl compounds using several catalysts..$^{8-10}$

For many years, chemical reactions in water have attracted the attention of chemists. ${ }^{11}$ From an environmental and economic point of view, water as a solvent or media has many advantages and usually results in excellent efficiency and selectivity. ${ }^{12}$ Accordingly, we describe an ecofriendly method for the synthesis of some new and known dicoumarols containing an aryloyl group in water as solvent.

\section{Results and Discussion}

Recently, we have been involved in studies involving the synthesis of new coumarin derivatives. ${ }^{13}$ In this regard, we found that the condensation between 4-hydroxycoumarin (1) and aryl glyoxals 2 in the presence of catalytic amounts of $p$-toluenesulfonic acid ( $p$-TSA) in water under reflux produces new and known dicoumarols 3 (Scheme 1). $p$-TSA is well known as catalyst because of its advantages, such as low corrosivity, simple handling and it is inexpensive. It has been widely used as an efficient catalyst in several organic reactions. ${ }^{14-16}$

In order to establish the best conditions for the synthesis of 3 using $p$-TSA as catalyst, reaction between 4-hydroxycoumarin (1) and phenyl glyoxal was selected as a model. Results indicated

* To whom correspondence should be addressed. E-mail: saeidkhm @ yahoo.com / khalileskandari@yahoo.com

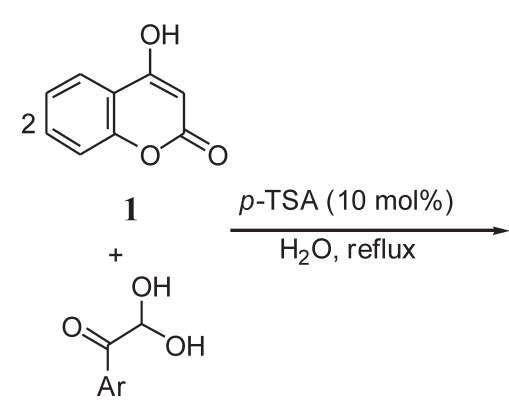

2

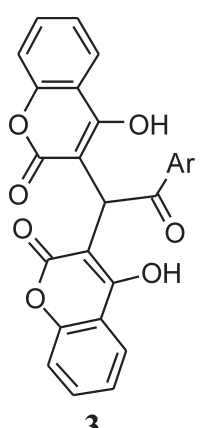

3
Scheme 1

Synthesis of dicoumarols catalyzed by $p$-TSA.

that the reaction did not go to completion in the absence of catalyst even after extended reaction times. Higher loadings of catalyst did not afford a marked influence on the product yield nor reaction rate. In another experiment, in order to illustrate the effect of solvent or media on the reaction progress, several different solvents were employed, the results of which are illustrated in Table 1.

It may be concluded that protonic solvents such as $\mathrm{EtOH}$, $\mathrm{MeOH}$, and $\mathrm{H}_{2} \mathrm{O}$ can accelerate the condensation reaction. Finally, it was found that this reaction is enhanced using $p$-TSA $(10 \mathrm{~mol} \%)$ as catalyst under reflux in $\mathrm{H}_{2} \mathrm{O}$ in $70 \mathrm{~min}$.

After determining the optimal reaction conditions, attention was focused on the extension of the scope of the method. For this, various aryl glyoxals 2 and 4-hydroxycoumarin (1) were reacted. Results are given in Table 2 in which it is apparent that aryl glyoxals, including those bearing electron-poor and electron-rich substituents, were able to undergo this reaction. Compared with a previously reported method which has used $\mathrm{AcOH}$ as reaction media, ${ }^{9}$ the present method provides environmentally safe conditions using water as solvent and $p$-TSA as catalyst to obtain the desired products with better yields than previous reported. Recently, organic synthesis on water has also been reviewed by Fokin and co-workers. ${ }^{17}$ Based on their study, it would appear that this reaction type may be placed in the category of 'on-water' synthetic reactions.

Based on the common mechanistic pathway of the Knoevenagel 
Table 1 Effect of catalyst amount and various solvents on the synthesis of $\mathbf{3 a}$ at reflux temperature.

\begin{tabular}{|c|c|c|c|c|}
\hline Entry & Catalyst amount $/ \mathrm{mol} \%$ & Solvent & Time/min & Yield/\%* \\
\hline 1 & 10 & $\mathrm{MeOH}(50 \mathrm{~mL})$ & 50 & 78 \\
\hline 2 & 10 & $\mathrm{EtOH}(50 \mathrm{~mL})$ & 45 & 80 \\
\hline 3 & 10 & THF (50 mL) & 50 & 75 \\
\hline 4 & 10 & $\mathrm{CH}_{2} \mathrm{Cl}_{2}(50 \mathrm{~mL})$ & 120 & 50 \\
\hline 5 & 10 & $\mathrm{EtOH} / \mathrm{H}_{2} \mathrm{O}(1 / 1)(50 \mathrm{~mL})$ & 60 & 82 \\
\hline 6 & 10 & $\mathrm{H}_{2} \mathrm{O}(50 \mathrm{~mL})$ & 70 & 80 \\
\hline 7 & - & $\mathrm{H}_{2} \mathrm{O}(50 \mathrm{~mL})$ & 180 & 30 \\
\hline 8 & 5 & $\mathrm{H}_{2} \mathrm{O}(50 \mathrm{~mL})$ & 180 & 75 \\
\hline 9 & 20 & $\mathrm{H}_{2} \mathrm{O}(50 \mathrm{~mL})$ & 50 & 77 \\
\hline
\end{tabular}

* Values provided are the average of three experiments.

Table 2 Synthesis of dicoumarols using $p$-TSA $(10 \mathrm{~mol} \%)$ under reflux in $\mathrm{H}_{2} \mathrm{O}$

\begin{tabular}{|c|c|c|c|c|}
\hline Entry & Ar & Time (min) & Yield $/ \%{ }^{a}$ [Lit.] & $\mathrm{Mp} /{ }^{\circ} \mathrm{C}$ [Lit.] \\
\hline $3 a$ & $\mathrm{C}_{6} \mathrm{H}_{5}$ & 70 & $82(74)^{9}$ & $\begin{array}{c}197-199 \\
(200-202)^{9}\end{array}$ \\
\hline $3 b$ & $4-\mathrm{F}-\mathrm{C}_{6} \mathrm{H}_{4}$ & 65 & 78 & $273-235$ \\
\hline $3 c$ & $4-\mathrm{Br}-\mathrm{C}_{6} \mathrm{H}_{4}$ & 70 & $81(79)^{9}$ & $\begin{array}{c}240-242 \\
(236-238)^{9}\end{array}$ \\
\hline $3 d$ & $4-\mathrm{NO}_{2}-\mathrm{C}_{6} \mathrm{H}_{4}$ & 60 & $80(76)^{9}$ & $\begin{array}{l}243-245 \\
(240-242)^{9}\end{array}$ \\
\hline $3 e$ & $4-\mathrm{MeO}-\mathrm{C}_{6} \mathrm{H}_{4}$ & 55 & 78 & $265-267$ \\
\hline $3 f$ & 3-MeO- $\mathrm{C}_{6} \mathrm{H}_{4}$ & 75 & 70 & $205-207$ \\
\hline $3 g$ & $4-\mathrm{Cl}-\mathrm{C}_{6} \mathrm{H}_{4}$ & 70 & 75 & $250-252$ \\
\hline $3 h$ & & 60 & 84 & $255-257$ \\
\hline
\end{tabular}

a Isolated yields.

and Michael reaction, ${ }^{18,19}$ we propose a reasonable mechanism involving the protonic acid-catalyzed reaction of aryl glyoxal 2 with 4-hydroxycoumarin (1), as depicted in Scheme 2. Firstly,
Knoevenagel condensation between 4-hydroxycoumarin (oxonium ions not depicted in mechanism) and the aryl glyoxal generates the non-isolable $\alpha, \beta$-unsaturated carbonyl compound 4. Attack of the next 4-hydroxycoumarin molecule (1) through a Michael-type addition to 4 and subsequent, the enolization of adduct 5 , gives the final product 3 .

\section{Experimental}

\subsection{General}

All chemicals were purchased from Merck and Aldrich. Aryl gloxals were synthesized in accord with our previous method. ${ }^{20}$ The reactions were monitored by thin layer chromatography (TLC; silica-gel $60 \mathrm{~F}_{254}$, n-hexane: ethyl acetate). IR spectra were recorded on a FT-IR JASCO-680 and the ${ }^{1} \mathrm{H}$ NMR and ${ }^{13} \mathrm{C}$ NMR spectra were recorded on a Bruker Avance Ultra Shield spectrometer respectively at 400,300,100, and $75 \mathrm{MHz}$. The Vario EL-III CHNS elemental analyzer from Isfahan Industrial University was used for elemental analysis. The structures and purity of the products were deduced from their IR, elemental analysis, and NMR spectral data.

\subsection{Preparation of Dicoumarols 3}

A mixture of 4-hydroxycoumarin 1 (20 mmol, $3.2 \mathrm{~g})$, aryl glyoxals $2(10 \mathrm{mmol})$ and $p$-TSA $(10 \mathrm{~mol} \%)$ in $\mathrm{H}_{2} \mathrm{O}(50 \mathrm{~mL})$ was

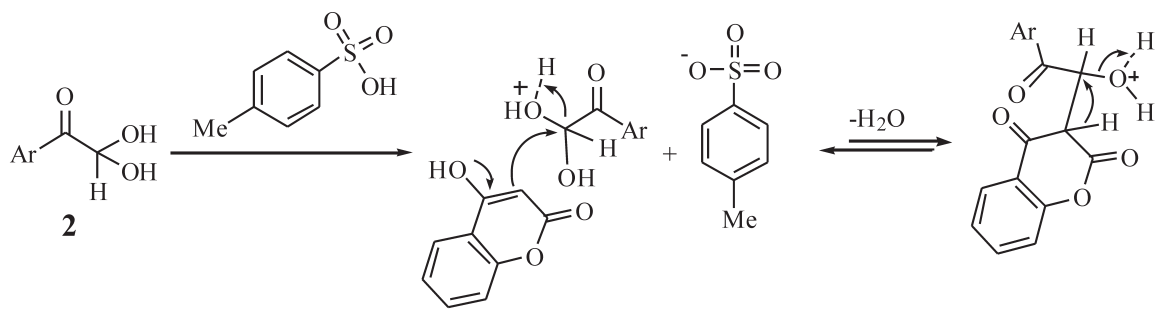

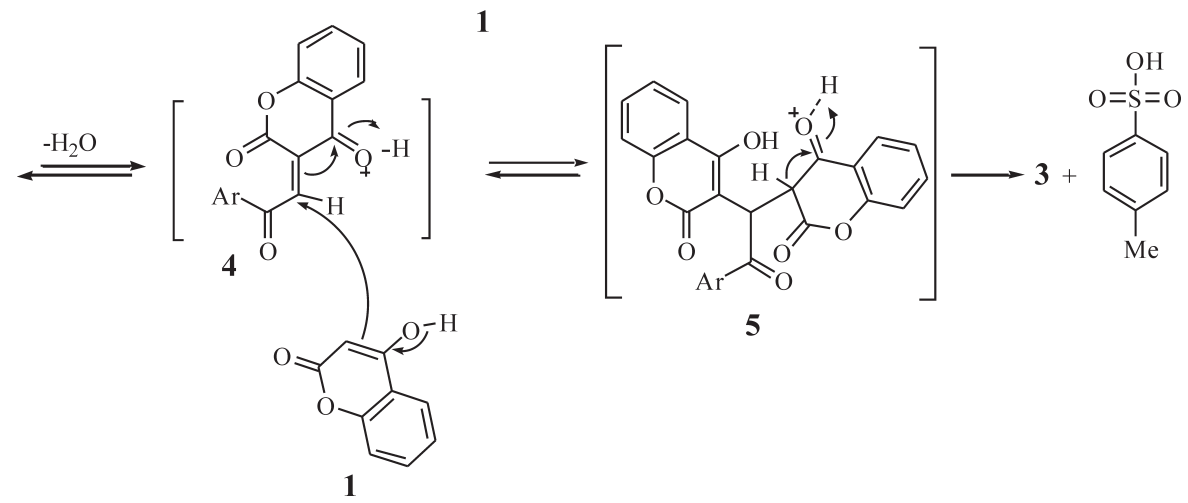

Scheme 2

Plausible mechanism for $p$-TSA-catalyzed condensation of 4-hydroxycoumarin with aryl glyoxal $\left(\mathrm{H}^{+}\right.$transfers not depicted). 
refluxed for an appropriate time mentioned in Table 2 . The progress of the reaction was monitored by TLC (EtOAc/hexane, 1:1). After completion, the mixture was poured on ice and the precipitate was filtered and purified by recrystallization from $\mathrm{EtOH} / \mathrm{THF}$ (2:1). In some cases, column chromatography is needed (EtOAc/hexane, 1:1).

Benzoyl[bis(4-hydroxycoumarin-3-yl)]methane (3a): Recrystallized from $\mathrm{EtOH} / \mathrm{THF}$ (TLC n-hexane:ethyl acetate, 1:1, Rf = 0.12); M.p. $197-199{ }^{\circ} \mathrm{C}$ ( Lit. $\left.^{9} 200-202{ }^{\circ} \mathrm{C}\right)$; IR (KBr) $v=3400-2900,3073$, 1698, 1659, 1618, 1566, 1271, $1100 \mathrm{~cm}^{-1} ;{ }^{1} \mathrm{H}$ NMR (DMSO-d $400 \mathrm{MHz}): \delta=10.95(\mathrm{~s}, 2 \mathrm{H}), 7.92\left(\mathrm{dd}, 2 \mathrm{H}, J_{1}=7.8, J_{2}=2.8 \mathrm{~Hz}\right)$, $7.63-7.58(\mathrm{~m}, 2 \mathrm{H}), 7.43(\mathrm{~d}, 2 \mathrm{H}, J=7.2 \mathrm{~Hz}), 7.39-7.31(\mathrm{~m}, 7 \mathrm{H}), 6.31$ (s, $1 \mathrm{H})$.

4-Flourobenzoyl[bis(4-hydroxycoumarin-3-yl)]methane (3b): Recrystallized from EtOH/THF (TLC n-hexane:ethyl acetate, 1:1, Rf = 0.15);M.p. $235-237^{\circ} \mathrm{C}$; IR (KBr) $v=3500-3300,3066,2887,1695$, 1650, 1619, 1600, 1567, 1271, 1225, $1107 \mathrm{~cm}^{-1} ;{ }^{1} \mathrm{H} \mathrm{NMR}\left(\mathrm{CDCl}_{3}\right.$, $300 \mathrm{MHz}): \delta=11.15(\mathrm{~s}, 2 \mathrm{H}), 7.89\left(\mathrm{dd}, 2 \mathrm{H}, J_{1}=8.2, J_{2}=1.6 \mathrm{~Hz}\right)$ $7.79-7.75(\mathrm{~m}, 2 \mathrm{H}), 7.56-7.50(\mathrm{~m}, 2 \mathrm{H}), 7.33-7.24(\mathrm{~m}, 4 \mathrm{H}), 6.94(\mathrm{t}, 2 \mathrm{H}$ $J=8.6 \mathrm{~Hz}) .{ }^{13} \mathrm{C} \mathrm{NMR}\left(\mathrm{CDCl}_{3}, 75 \mathrm{MHz}\right): \delta=192.9,165.4,152.4$, $133.2,132.0,130.7,130.6,125.0,124.5,116.7,116.3,115.9,115.6$, 42.8. Anal. Calcd. for $\mathrm{C}_{26} \mathrm{H}_{15} \mathrm{FO}_{7}: \mathrm{C}, 68.12 ; \mathrm{H}, 3.30$. Found: $\mathrm{C}, 68.30$; $\mathrm{H}, 3.22$.

4-Bromobenzoyl[bis(4-hydroxycoumarin-3-yl)]methane (3c): Recrystallized from EtOH/THF (TLC n-hexane:ethyl acetate, 1:1, Rf = 0.11); M.p. $240-242{ }^{\circ} \mathrm{C}$ (Lit. $\left.{ }^{9} 236-238^{\circ} \mathrm{C}\right) ; \mathrm{IR}(\mathrm{KBr}) v=3400-2900$, $1711,1651,1614,1564,1497,1267,1099 \mathrm{~cm}^{-1},{ }^{1} \mathrm{H}$ NMR (DMSO-d $d_{6}$ $400 \mathrm{MHz}): \delta=10.56(\mathrm{~s}, 2 \mathrm{H}), 7.89(\mathrm{~d}, 2 \mathrm{H}, J=7.6 \mathrm{~Hz}), 7.59(\mathrm{t}, 2 \mathrm{H}, J=$ $7.6 \mathrm{~Hz}), 7.40-7.29(\mathrm{~m}, 6 \mathrm{H}), 7.11(\mathrm{~d}, 2 \mathrm{H}), 6.28(\mathrm{~s}, 1 \mathrm{H})$.

4-Nitrobenzoyl[bis(4-hydroxycoumarin-3-yl)]methane (3d): Recrystallized from EtOH/THF (TLC n-hexane:ethyl acetate, 1:1, Rf = 0.12); M.p. $243-245^{\circ} \mathrm{C}$ (Lit. $\left.{ }^{9} 240-242{ }^{\circ} \mathrm{C}\right) ; \mathrm{IR}(\mathrm{KBr}) v=3400-2900$, 2883, 1715, 1650, 1614, 1565, 1518, 1341, 1266, $1102 \mathrm{~cm}^{-1} ; 10.95$ (s, $2 \mathrm{H}), 7.92\left(\mathrm{dd}, 2 \mathrm{H}, J_{1}=7.8 \mathrm{~Hz}, J_{2}=2.8 \mathrm{~Hz}\right), 7.63-7.58(\mathrm{~m}, 2 \mathrm{H}), 7.43$ $(\mathrm{d}, 2 \mathrm{H}, J=7.2 \mathrm{~Hz}), 7.39-7.31(\mathrm{~m}, 7 \mathrm{H}), 6.31(\mathrm{~s}, 1 \mathrm{H})$.

4-Methoxybenzoyl[bis(4-hydroxycoumarin-3-yl)]methane (3e): Purified by column chromatography (EtOAc/hexane, 1:1) (TLC n-hexane:ethyl acetate, 1:1, Rf = 0.16); M.p. $265-267^{\circ} \mathrm{C}$; IR (KBr) $v=35003300,3076,2978,1684,1650,1620,1601,1571,1263 \chi \mu^{-1}$; ${ }^{1} \mathrm{H}$ NMR $\left(\mathrm{CDCl}_{3}, 300 \mathrm{MHz}\right): \delta=11.22(\mathrm{~s}, 2 \mathrm{H}), 8.00\left(\mathrm{dd}, 2 \mathrm{H}, \mathrm{J}_{1}=\right.$ $\left.8.2, J_{2}=1.6 \mathrm{~Hz}\right), 7.77-7.72(\mathrm{~m}, 2 \mathrm{H}), 7.55-7.49(\mathrm{~m}, 2 \mathrm{H}), 7.32-7.24$ $(\mathrm{m}, 4 \mathrm{H}), 6.77-6.72(\mathrm{~m}, 2 \mathrm{H}), 6.00(\mathrm{~s}, 1 \mathrm{H}), 3.71(\mathrm{~s}, 3 \mathrm{H}) ;{ }^{13} \mathrm{C} \mathrm{NMR}$ $\left(\mathrm{CDCl}_{3}, 75 \mathrm{MHz}\right): \delta=193.1,165.2,163.5,152.4,133.0,130.4,128.3$, $124.9,124.5,116.6,116.4,113.8,55.4,42.6$. Anal. Calcd. for $\mathrm{C}_{27} \mathrm{H}_{18} \mathrm{O}_{8}: \mathrm{C}, 68.94 ; \mathrm{H}, 3.86$. Found: $\mathrm{C}, 69.10 ; \mathrm{H}, 3.69$.

3-Methoxybenzoyl[bis(4-hydroxycoumarin-3-yl)]methane (3f): Purified by column chromatography (EtOAc/hexane, 1:1) (TLCn-hexane:ethyl acetate, 1:1, Rf = 0.15); M.p. 205-207 ${ }^{\circ} \mathrm{C}$; IR (KBr) $v=3500-3300,1693,1655,1619,1602,1567,1273,1427 \mathrm{~cm}^{-1}$; ${ }^{1} \mathrm{H}$ NMR $\left(\mathrm{CDCl}_{3}, 300 \mathrm{MHz}\right): \delta=11.16(\mathrm{~s}, 1 \mathrm{H}), 8.00\left(\mathrm{dd}, 2 \mathrm{H}, \mathrm{J}_{1}=\right.$ $\left.8.2, J_{2}=1.6 \mathrm{~Hz}\right), 7.55-7.49(\mathrm{~m}, 2 \mathrm{H}), 7.34-7.24(\mathrm{~m}, 6 \mathrm{H}), 7.12(\mathrm{t}, 1 \mathrm{H}$, $J=8.2 \mathrm{~Hz}), 6.94-6.90(\mathrm{~m}, 1 \mathrm{H}), 6.00(\mathrm{~s}, 1 \mathrm{H}), 3.69(\mathrm{~s}, 3 \mathrm{H}) ;{ }^{13} \mathrm{C} \mathrm{NMR}$ $\left(\mathrm{CDCl}_{3}, 75 \mathrm{MHz}\right): \delta=194.2,165.2,159.7,152.4,136.9,133.1,129.4$, 125.0, 124.5, 120.2, 120.1, 116.7, 116.4, 112.4, 42.9. Anal. Calcd. for $\mathrm{C}_{27} \mathrm{H}_{18} \mathrm{O}_{8}: \mathrm{C}, 68.94 ; \mathrm{H}, 3.86$. Found: $\mathrm{C}, 69.06 ; \mathrm{H}, 3.65$.

4-Chlorobenzoyl[bis(4-hydroxycoumarin-3-yl)]methane (3g): Purified by column chromatography (EtOAc/hexane, 1:1) (TLC nhexane:ethyl acetate, 1:1, Rf =0.18); M.p. $250-252{ }^{\circ} \mathrm{C}$; IR (KBr) $v=3500-3300,3080,2884,1713,1665,1650,1614,1564,1266,1090$, $767 \mathrm{~cm}^{-1} ;{ }^{1} \mathrm{HNMR}\left(\mathrm{DMSO}-d_{6}, 400 \mathrm{MHz}\right): \delta=11.10(\mathrm{~s}, 2 \mathrm{H}), 7.85(\mathrm{~d}$,
$2 \mathrm{H}, J=6.0 \mathrm{~Hz}), 7.72(\mathrm{~d}, 2 \mathrm{H}, J=5.2 \mathrm{~Hz}), 7.62-7.52(\mathrm{~m}, 4 \mathrm{H})$, 7.31-7.25 (m, 4H), 6.28 (s, 1H); ${ }^{13} \mathrm{C}$ NMR (DMSO- $\left.d_{6}, 100 \mathrm{MHz}\right)$ : $\delta=196.1,165.9,163.3,152.2,135.9,131.6,131.2,129.3,125.9,123.8$, 123.4, 118.0, 115.8, 101.6, 42.9. Anal. Calcd. for $\mathrm{C}_{26} \mathrm{H}_{15} \mathrm{ClO}_{7}$ : C, 65.76; H, 3.18. Found: C, 65.91; H, 3.03.

2-Naphthoyl[bis(4-hydroxycoumarin-3-yl)]methane (3h): Recrystallized from EtOH/THF (TLC n-hexane:ethyl acetate, 1:1, Rf = 0.11); M.p. 255-257 ${ }^{\circ} \mathrm{C}$; IR (KBr) $v=3550-3300,1694,1653,1617$, $1565,1454,1280 \mathrm{~cm}^{-1} ;{ }^{1} \mathrm{H}$ NMR $\left(\mathrm{CDCl}_{3}, 300 \mathrm{MHz}\right): \delta=11.24(\mathrm{~s}$, $2 \mathrm{H}), 8.27(\mathrm{~s}, 1 \mathrm{H}), 8.01\left(\mathrm{dd}, 2 \mathrm{H}, J_{1}=8.2, J_{2}=1.6 \mathrm{~Hz}\right), 7.83-7.72(\mathrm{~m}$, $4 \mathrm{H}), 7.54-7.43(\mathrm{~m}, 4 \mathrm{H}), 7.33-7.23(\mathrm{~m}, 4 \mathrm{H}), 6.19(\mathrm{~s}, 1 \mathrm{H}) .{ }^{13} \mathrm{C} \mathrm{NMR}$ (DMSO- $\left.d_{6}, 75 \mathrm{MHz}\right): \delta=177.3,166.6,163.6,152.3,134.4,134.3$, $131.8,131.5,129.1,127.9,127.5,126.7,124.1,123.9,123.3,118.5$, 115.7, 101.6, 43.1. Anal. Calcd. for $\mathrm{C}_{30} \mathrm{H}_{18} \mathrm{O}_{7}: \mathrm{C}, 73.47 ; \mathrm{H}, 3.70$. Found: C, 73.68; H, 3.75.

\section{Conclusion}

An improved route for the synthesis of dicoumarols containing an aryloyl group from simple substrates and $p$-TSA catalyst has been achieved with a very high atom economy for the preparation of pharmaceutically relevant heterocyclic systems. Importantly, use of water as a cheap and clean media for reaction should place this chemistry in the category of Green Chemistry. A total of five new compounds were obtained.

\section{Supplementary material}

The ${ }^{1} \mathrm{H}$ and ${ }^{13} \mathrm{C}$ spectra of all the novel compounds are given in the online supplement.

\section{Acknowledgement}

The authors are grateful to Yasouj University for partial support.

\section{References}

1 H.A. Campbell and K.P. Link, Studies on the hemorrhagic sweet clover disease: IV. The isolation and crystallization of the hemorrhagic agent, J. Biol. Chem., 1941, 138, 21-33.

2 Z. Karimi-Jaberi and L. Zarei, Synthesis of coumarins and 2,3-dihydroquinazolin-4(1H)-ones using trichloroacetic acid as a catalyst, Acta Chim. Slov., 2013, 60, 178-183

3 K.V. Sashidhara, G.R. Palnati, S.R. Avula, and A. Kumar, Efficient and general synthesis of 3-aryl coumarins using cyanuric chloride, Synlett., 2012, 23, 611-621.

4 A. Barzegar, M.D. Davari, N. Chaparzadeh, N. Zarghami, J.Z. Pedersen, S. Incerpi, L. Saso and A.A. Moosavi-Movahedi, Theoretical and experimental studies on the structure-antioxidant activity relationship of synthetic 4-methylcoumarins, J. Iran. Chem. Soc., 2011, 8, 973-982.

5 J. Ferguson, F. Zeng, and H. Alper, Palladium-catalyzed oxidative cyclocarbonylation of 2-vinylphenols enables a direct synthesis of various coumarins in good yields in the presence of low pressures of CO, and air or 1,4-benzoquinone as the oxidant, Org. Lett., 2012, 14, 5602-5605.

6 S.R. Cherkupally and R. Mekala, Synthesis of novel 6,6'-methylenebis-[3-(2-anilinoacetyl)-4-hydroxycoumarin] derivatives, Chem. Pharm. Bull., 2008, 56, 1732-1734.

7 D.M. Bellis, M.S. Spring and J.R. Stoker, The biosynthesis of dicoumarol, 1967, Biochem. J., 103, 202-206.

8 S. Khodabakhshi and M. Baghernejad, Silica gel: a very cheap, reusable, and efficient catalyst for green preparation of some bis-4hydroxycoumarin on water, Iran. J. Catal., 2013, 3, 67-71.

9 N. Kolos, L.L. Gozalishvili and F.G. Yaremenko, Aroylbis(4-hydroxycoumarin-3-yl)methanes in reactions with 1,2-diaminobenzenes, Russ. Chem. Bull., 2007, 56, 2277-2283.

10 G.M. Ziarani and P. Hajiabbasi, Recent application of 4-hydroxycoumarin in multi-component reactions, Heterocycles, 2013, 87, 1415-1439.

11 A. Podgorseka, S. Stavbera, M. Zupana and J. Iskra, Free radical 
S. Afr. J. Chem., 2015, 68, 53-56,

$<$ http://journals.sabinet.co.za/sajchem/>.

bromination by the $\mathrm{H}_{2} \mathrm{O}_{2}-\mathrm{HBr}$ system on water, Tetrahedron Lett., 2006, 47, 7245-7247.

12 B. Karami, S.J. Hoseini, K. Eskandari, A. Ghasemi and H. Nasrabadi, Synthesis of xanthene derivatives by employing $\mathrm{Fe}_{3} \mathrm{O}_{4}$ nanoparticles as an effective and magnetically recoverable catalyst in water, Catal. Sci. Technol., 2012, 2, 331-338.

13 S. Khodabakhshi, B. Karami and K. Eskandari, Titanium dioxide nanowires as green and heterogeneous catalysts for the synthesis of novel pyranocoumarins, C. R. Chimie, 2014, 17, 35-40.

14 G. Le Bras, A. Hamze, S. Messaoudi, O. Provot, P.-B. Le Calvez, J.-D. Brion and M. Alami, Synthesis of isocoumarin via $p$ TSA-catalyzed annulation of diarylalkynes, Synthesis, 2008, 1607-1611.

15 C.R.B. da Silva, V.L.C. Gonçalves, E.R. Lachter and C.J.A. Mota, Etherification of glycerol with benzyl alcohol catalyzed by solid acids, J. Braz. Chem. Soc., 2009, 20, 201-204.

16 A. Mobinikhaledi, H. Moghanian and M. Deinavizadeh, $p$ TSA-cata- lyzed condensation of xylenols and aldehydes under solvent-free conditions: one-pot synthesis of $9 \mathrm{H}$-xanthene or bisphenol derivatives, C. R. Chimie, 2013, 16, 1035-1041.

17 A. Chanda and V.V. Fokin, Organic synthesis "on water" Chem. Rev., 2009, 109, 725-748.

18 M.M. Heravi, S. Sadjadi, N. Mokhtari Haj, H.A. Oskooie, F.F. Bamoharram, Role of various heteropolyacids in the reaction of 4-hydroxycoumarin, aldehydes and ethylcyanoacetate, Catal. Commun., 2009, 10, 1643-1646.

19 Singh, P. Kumar, A. Katyal, R. Kalra, S.K. Dass, S. Prakash, R. Chandra, Phosphotungstic acid: an efficient catalyst for the aqueous phase synthesis of bis-(4-hydroxycoumarin-3-yl)methanes, Catal. Lett., 2010, 134, 303-308.

20 B. Karami, S. Khodabakhshi and M. Nikrooz, Synthesis of aza-polycyclic compounds: novel phenazines and quinoxalines using molybdate sulfuric acid (MSA), Polycyclic Aromat. Compds. 2011,31,97-109. 
Supplementary Information, S. Afr. J. Chem., 2015, 68, 53-56

A Facile and Practical $p$-Toluenesulfonic Acid Catalyzed Route to Dicoumarols Containing an Aroyl group

Saeed Khodabakhshi ${ }^{1, *}$, Bahador Karami ${ }^{2}$, Khalil Eskandari ${ }^{2, *}$ and Alimorad Rashidi ${ }^{1}$

${ }^{1}$ Nanotechnology Research Center, Research Institute of Petroleum Industry, Tehran, Iran

${ }^{2}$ Department of Chemistry, Yasouj University, P. O. Box 353, Yasouj, 75918-74831, Iran

\section{Table of contents}

${ }^{1} \mathrm{H}$ NMR and ${ }^{13} \mathrm{C}$ NMR spectra of products $\mathbf{3 b}, \mathbf{3 e}, \mathbf{3 f}, \mathbf{3 g}, \mathbf{3 h}$ 


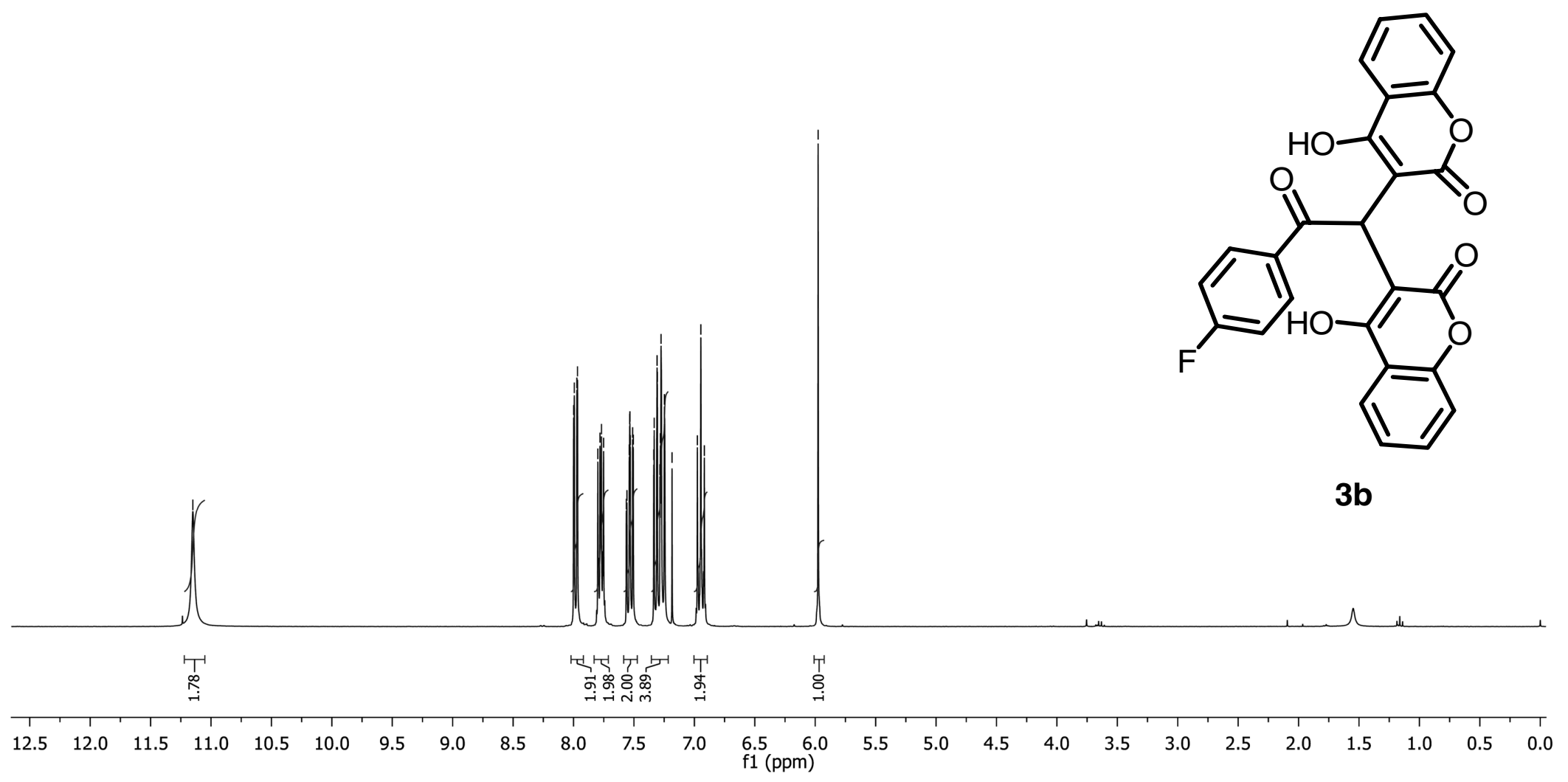

Figure $1{ }^{1} \mathrm{H}$ NMR spectrum of $\mathbf{3 b}$ 

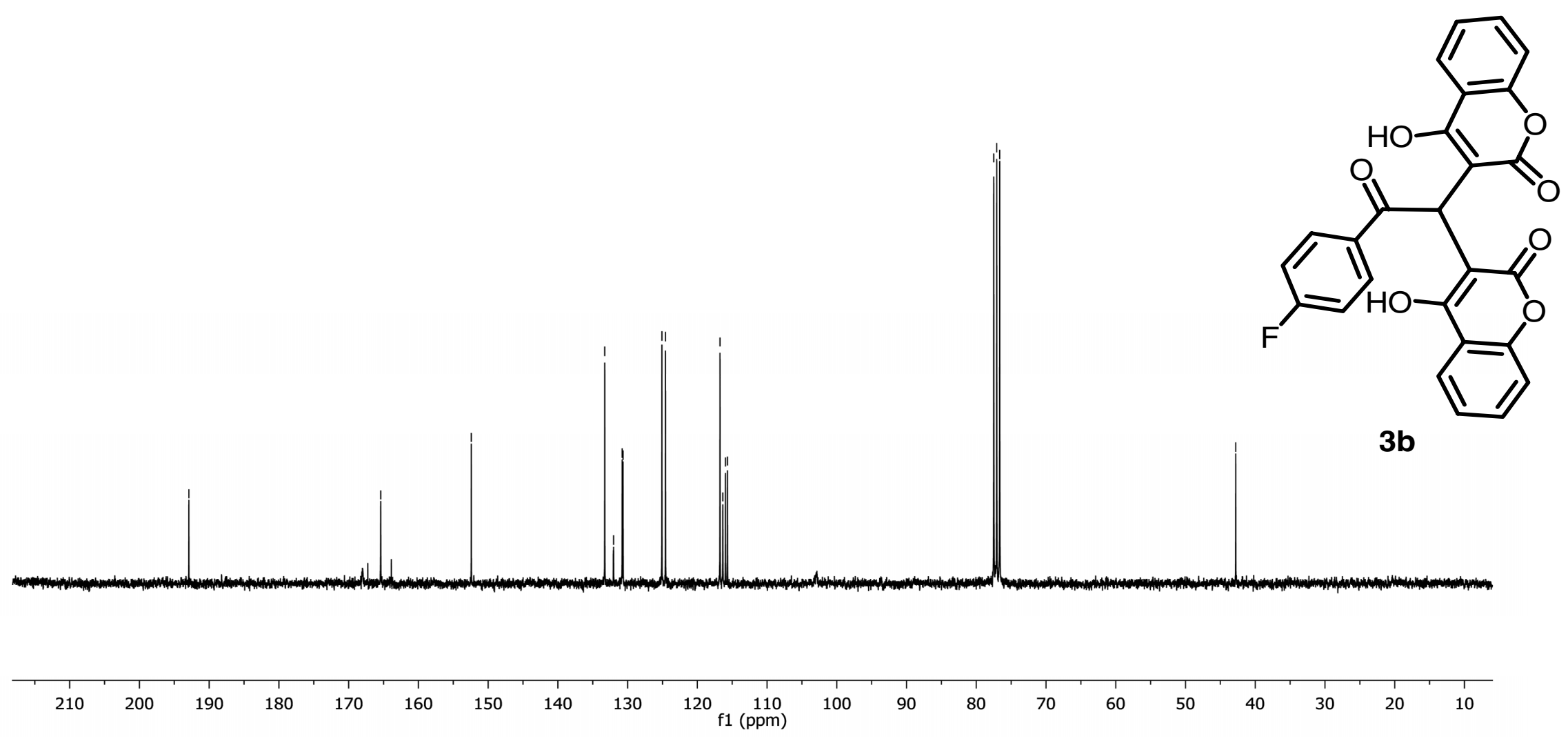

Figure $2{ }^{13} \mathrm{C}$ NMR spectrum of $\mathbf{3 b}$ 


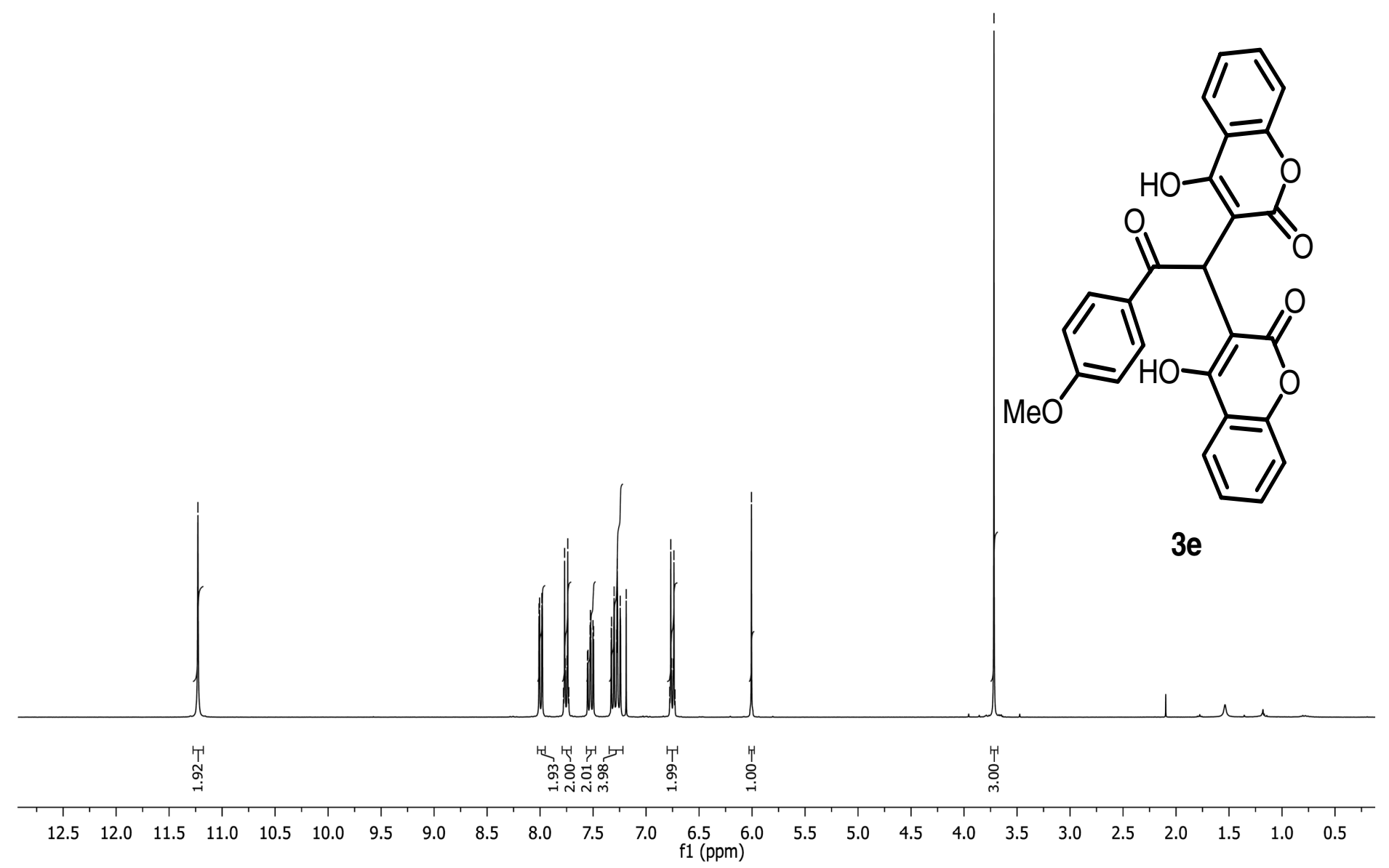

Figure $3{ }^{1} \mathrm{H}$ NMR spectrum of $\mathbf{3 e}$ 


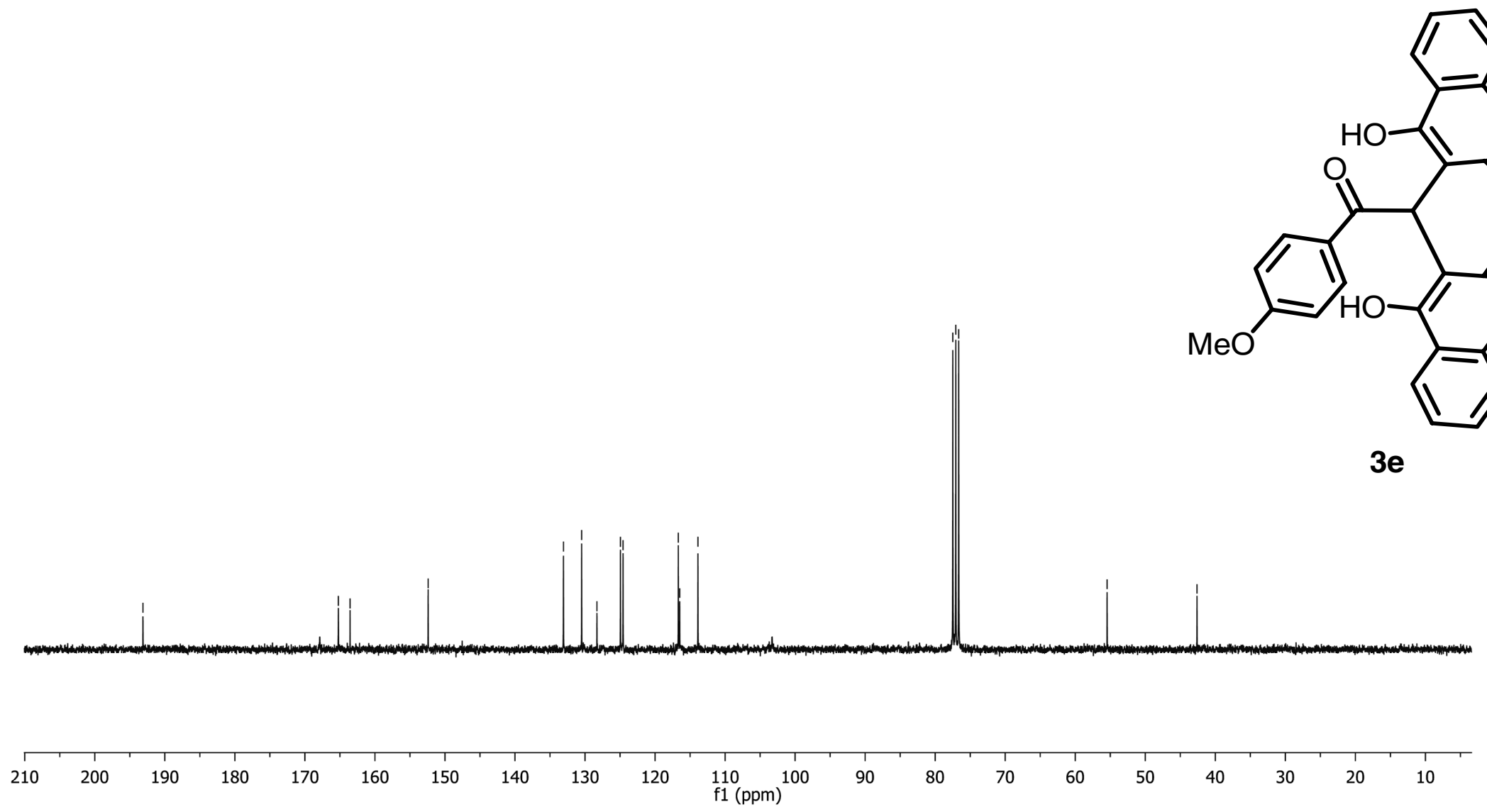

Figure $4{ }^{13} \mathrm{C}$ NMR spectrum of $\mathbf{3 e}$ 


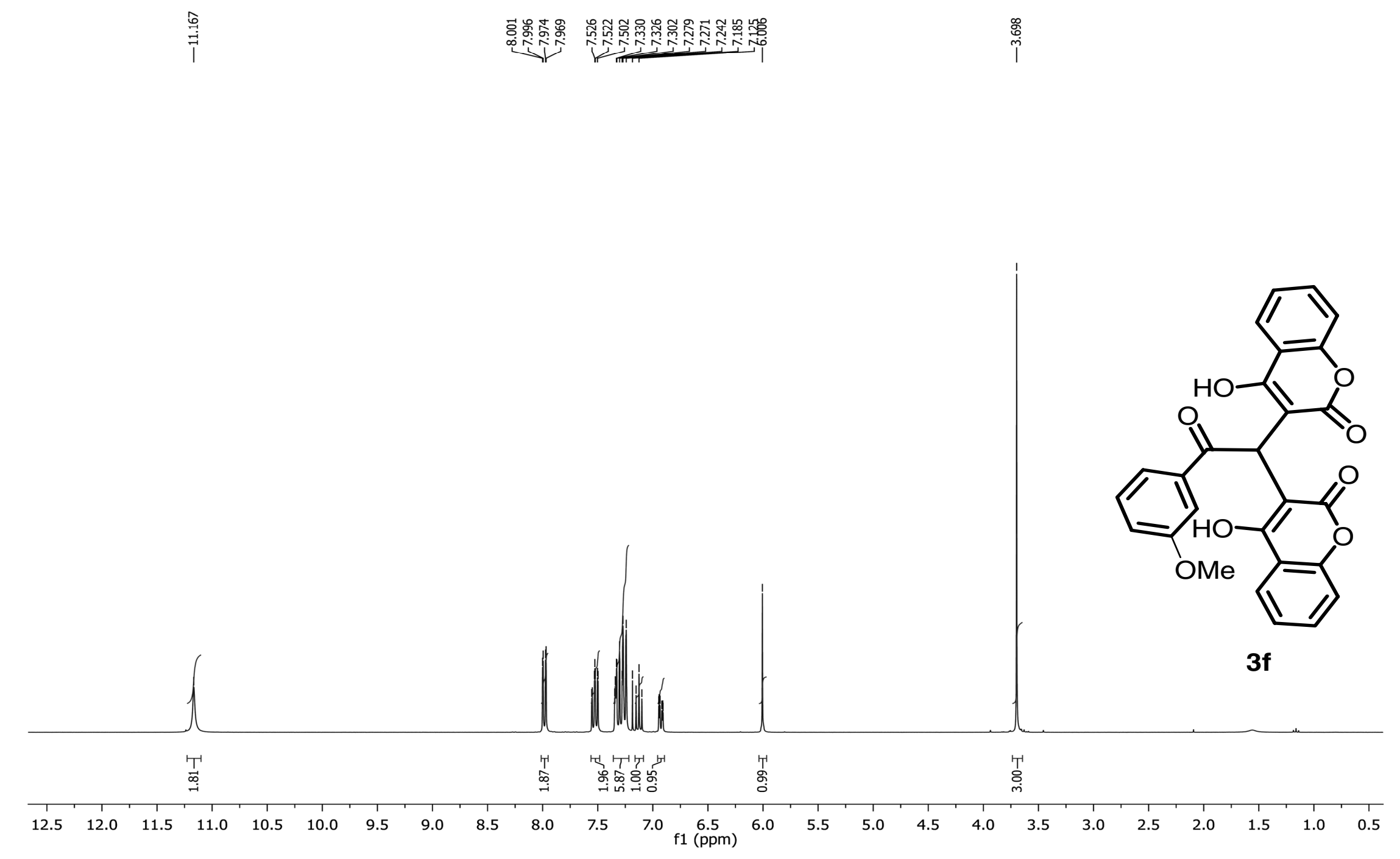

Figure $5{ }^{1} \mathrm{H}$ NMR spectrum of $\mathbf{3 f}$ 


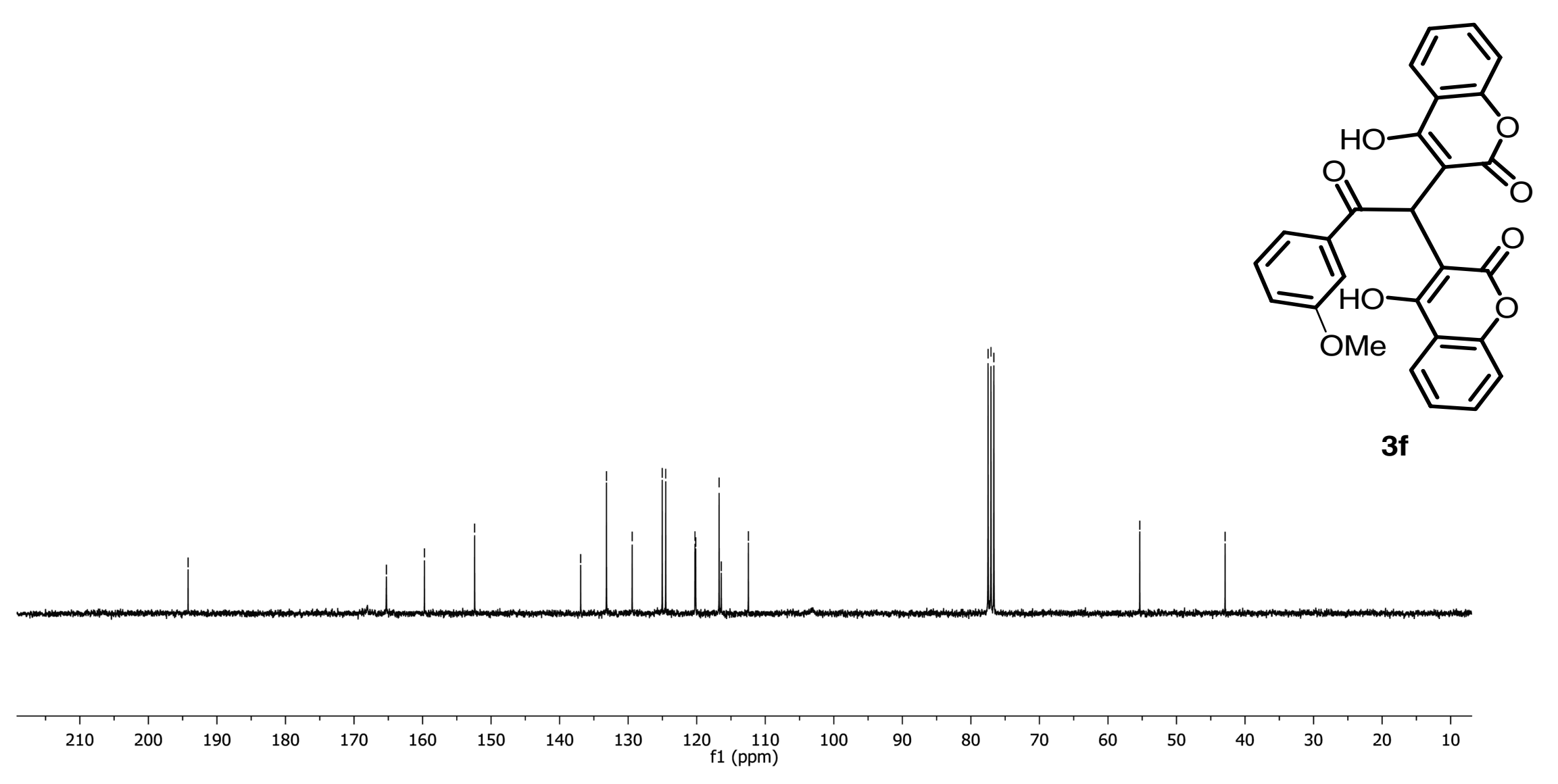

Figure $6{ }^{13} \mathrm{C}$ NMR spectrum of $\mathbf{3 f}$ 

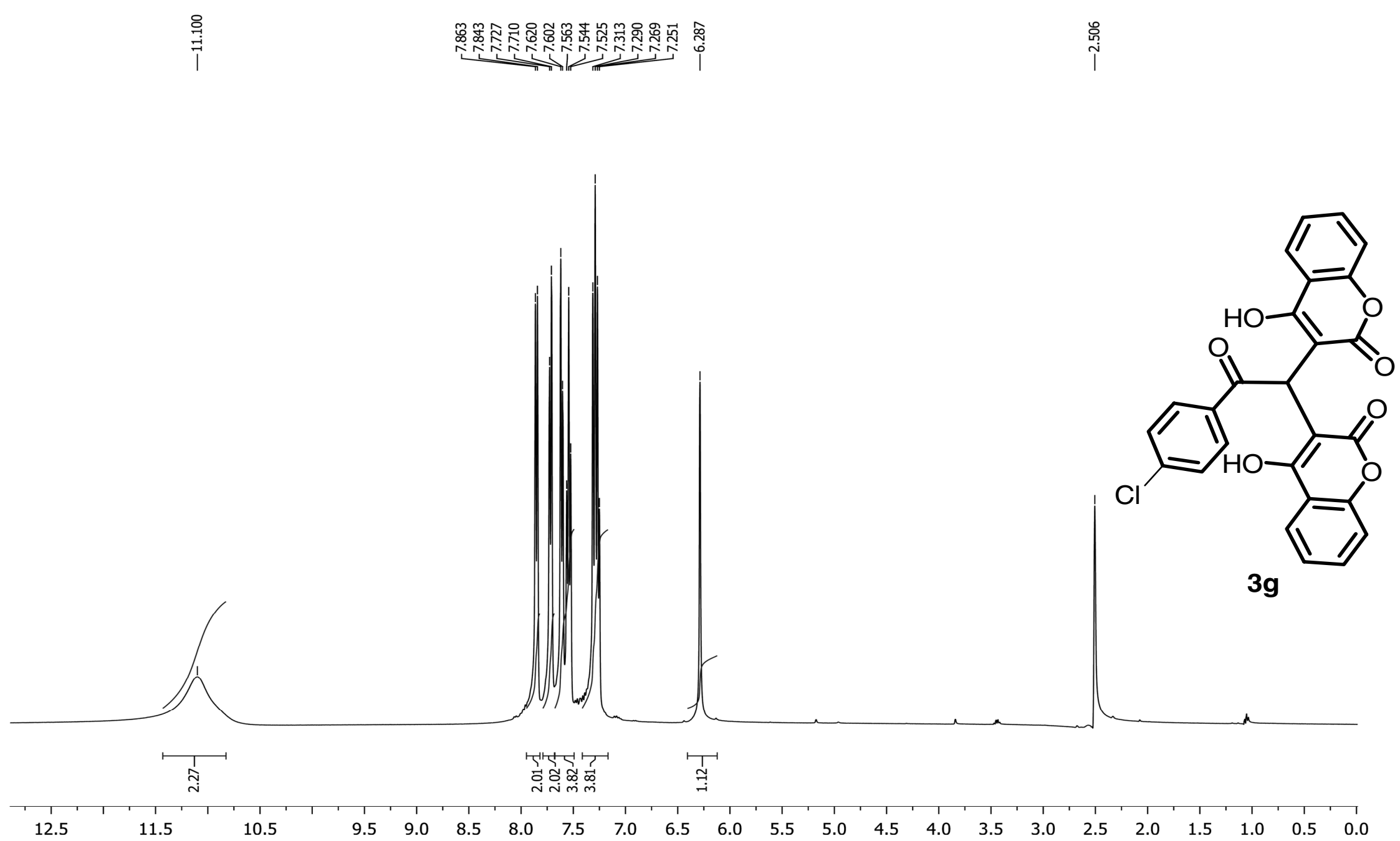

Figure $7{ }^{1} \mathrm{H}$ NMR spectrum of $\mathbf{3 g}$ 


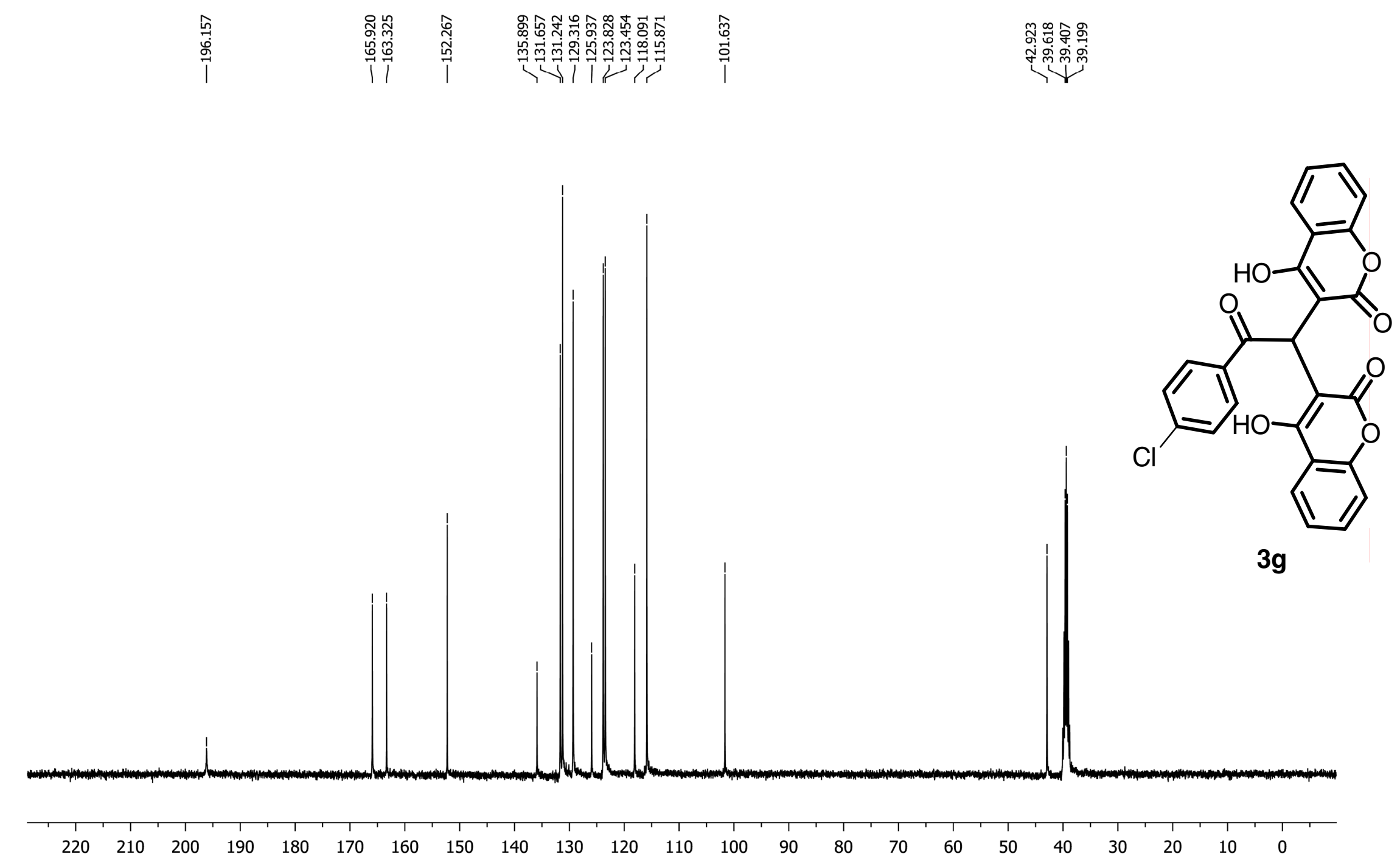

Figure $8{ }^{13} \mathrm{C}$ NMR spectrum of $\mathbf{3 g}$ 


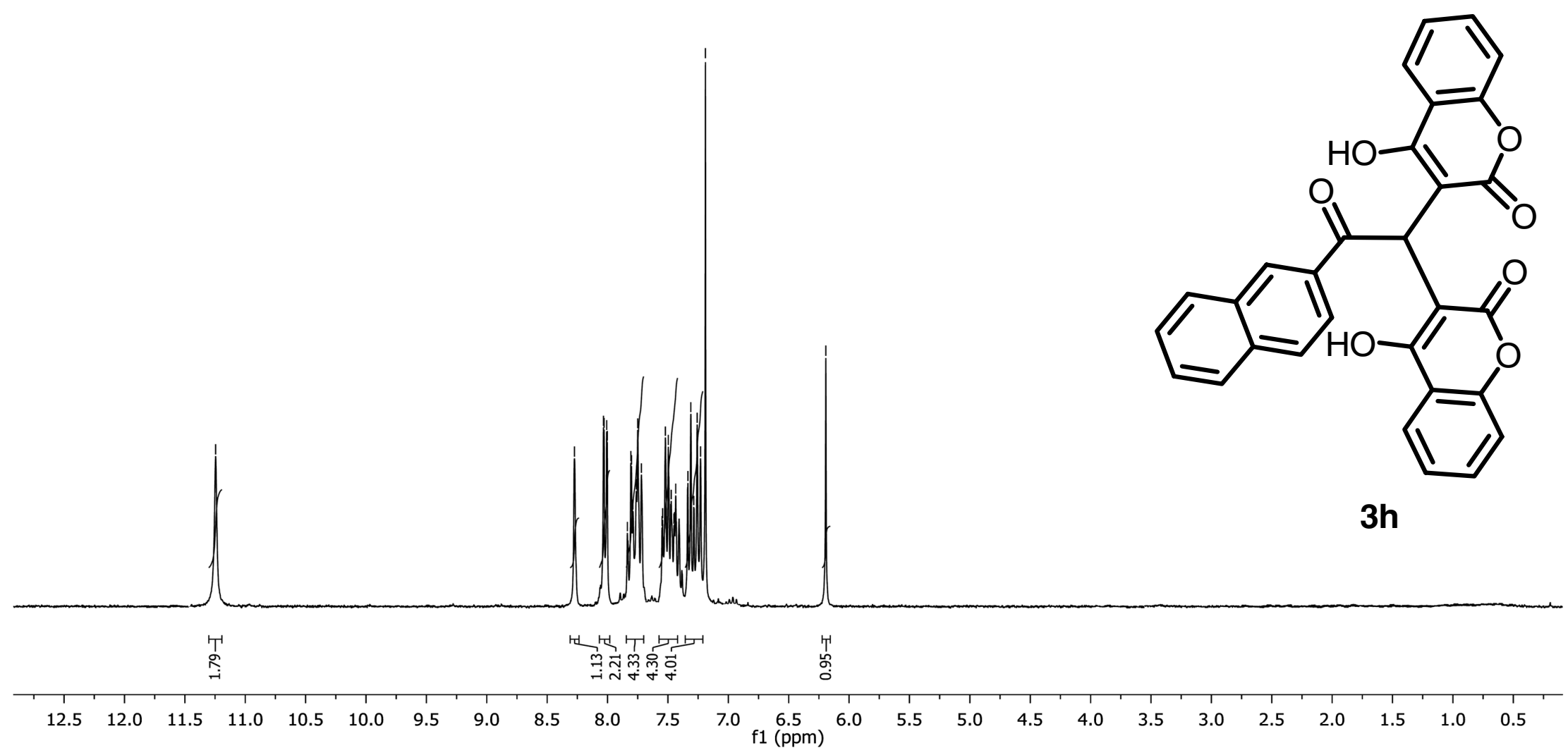

Figure $9{ }^{1} \mathrm{H}$ NMR spectrum of $\mathbf{3 h}$ 


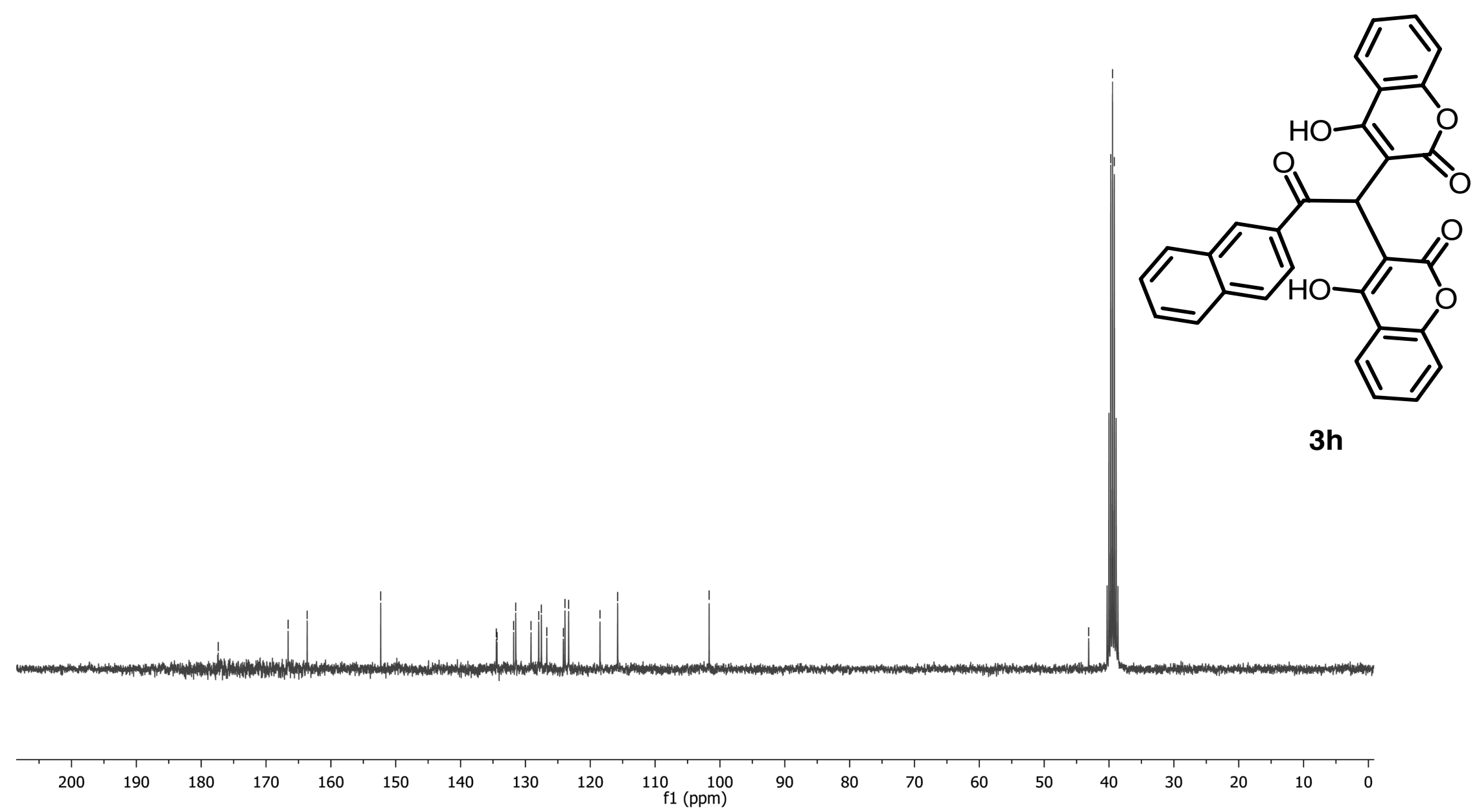

Figure $10{ }^{13} \mathrm{C}$ NMR spectrum of $\mathbf{3 h}$ 